Intermediate report on the project:

\title{
DETECTION IN URBAN SCENARIO USING COMBINED AIRBORNE IMAGING SENSORS
}

\author{
Ingmar Renhorn $^{(* a)}$, Maria Axelsson ${ }^{(a)}$, Koen Benoist ${ }^{(b)}$, Dirk Bourghys ${ }^{(c)}$, Yannick Boucher ${ }^{(d)}$, \\ Xavier Briottet $^{(\mathrm{d})}$, Sergio De Ceglie ${ }^{(\mathrm{e})}$, Rob Dekker ${ }^{(\mathrm{b})}$, Alwin Dimmeler ${ }^{(\mathrm{f})}$, Remco Dost ${ }^{(\mathrm{g})}$, Ola \\ Friman $^{(a)}$, Ingebjørg Kåsen ${ }^{(\mathrm{h})}$, Jochen Maerker ${ }^{(\mathrm{f})}$, Mark van Persie ${ }^{(\mathrm{g})}$, Salvatore Resta ${ }^{(\mathrm{e})}$, Piet \\ Schwering ${ }^{(b)}$, Michal Shimoni ${ }^{(c)}$, Trym Vegard Haavardsholm ${ }^{(h)}$ \\ ${ }^{a}$ Swedish Defence Research Agency, Sweden; ${ }^{b}$ TNO, The Netherlands; ${ }^{c}$ RMA, Belgium; \\ ${ }^{\mathrm{d}}$ ONERA, France; ${ }^{\mathrm{e}}$ CISAM, Italy; ${ }^{\mathrm{f}}$ Fraunhofer IOSB, Germany; ${ }^{\mathrm{g}} \mathrm{NLR}$, The Netherlands; \\ ${ }^{\mathrm{h}}$ FFI, Norway
}

\begin{abstract}
The EDA project "Detection in Urban scenario using Combined Airborne imaging Sensors" (DUCAS) is in progress. The aim of the project is to investigate the potential benefit of combined high spatial and spectral resolution airborne imagery for several defense applications in the urban area. The project is taking advantage of the combined resources from 7 contributing nations within the EDA framework. An extensive field trial has been carried out in the city of Zeebrugge at the Belgian coast in June 2011. The Belgian armed forces contributed with platforms, weapons, personnel (soldiers) and logistics for the trial. Ground truth measurements with respect to geometrical characteristics, optical material properties and weather conditions were obtained in addition to hyperspectral, multispectral and high resolution spatial imagery.
\end{abstract}

High spectral/spatial resolution sensor data are used for detection, classification, identification and tracking.

Keywords: hyperspectral, imagery, detection, classification, sensor fusion, tracking, high resolution imagery

\section{INTRODUCTION}

Due to the complexity of urban environments and human activities, the spatial and spectral characteristics of targets and backgrounds become more diverse which makes the detection and the classification of man-made objects very difficult using a single sensor with limited spectral or spatial resolution. In urban and industrial areas, spatially unresolved materials are difficult to discriminate based on spectral features alone. High spatial resolution sensors can be utilized to classify man-made objects from shape but is at the same time limited in capability to allow differentiation between materials [1-4].

From a military point of view, the past few years have highlighted the difficulty in monitoring the dynamics of the traffic in narrow streets which creates a complexity that is dramatically different from the rural environment. Day and night capability for security purposes in the urban scene is even more challenging. Tracking of individual vehicles in a complex traffic pattern or detection of specific individuals in a crowd is very difficult.

*ingmar.renhorn@foi.se; phone +46 13378263

Infrared Technology and Applications XXXVIII, edited by Bjørn F. Andresen, Gabor F. Fulop, Paul R. Norton, Proc. of SPIE Vol. 8353, 83530I - @ 2012 SPIE · CCC code: 0277-786X/12/\$18 · doi: 10.1117/12.921473 
In the latest conflicts in e.g. Iraq and Afghanistan, the following issues have been emphasized [5]:

- Significant improvements in sensor technology and systems will provide the warfighter with improved tools for supporting detection and classification of targets in the urban scene.

- Due to the complex nature of the urban environment and the amount of obscuration and occlusion in the urban scene, integrated sensing modes using, e.g. radio frequency, radar, hyperspectral, multispectral, infrared, seismic, acoustic, and magnetic sensors combined with data fusion, is needed in order to attain comprehensive situation awareness.

- Because no single sensor can produce a comprehensive urban-operating picture, new concepts and designs with intrinsic network-centric warfare capabilities is needed for the urban scenario. Network-centric warfare includes transmitting data in a timely fashion via communication links in machine-to-machine format and providing tools to augment data fusion.

- In order to facilitate the integration of sensor systems, each subsystem must be tested with respect to feature extraction requirements. The system functionality must be designed to simplify the understanding of the information.

The European programs CEPA JP8.10 (2003-2005) and HIPOD (2006, EDA) [6] highlighted the gain brought by spectral imagery to improve the detection and classification capability in a rural environment, but showed also the difficulty to identify targets based on low spatial resolution hyperspectral information in tactical scenarios. As a consequence, these programs pointed out the interest of combining high spatial resolution with high spectral resolution imagery to improve the process chain from detection to identification. There was also a need to extend the program to the urban landscape.

The Steering Board of the European Defense Agency approved on 16 December 2005 the joint research project entitled "Detection in Urban scenario using Combined Airborne imaging Sensors (DUCAS)". The objective of the project is to investigate the potential benefit of combined high spatial and spectral resolution airborne imagery for several defense applications in urban areas. The project includes experimental testing of these techniques in conditions relevant for these applications. The formal start of the four year program was in December 2009.

The project covers three main subjects:

- To determine the detection performance of difficult targets in urban environments using combined hyperspectral and high spatial resolution active and passive sensors, in comparison with conventional techniques.

- To evaluate the improvement of the situation awareness and surveillance capability in complex urban areas, using these combined technologies.

- To propose conceptual combined system design for those application areas where the benefit of combined hyperspectral and high spatial resolution active and passive sensors is clear.

The DUCAS project involves 7 countries and complementary research groups from leading European research organizations: RMA (BE), ONERA (FR), Fraunhofer-IOSB (GE), CISAM (IT), NLR and TNO (NL), FFI (NO), FOI (SE). The DUCAS members will take financial and technical advantage in this co-operation through sharing of facilities, knowledge and work. Sweden, through FOI, leads the DUCAS project.

This report will shortly describe the background and the outcome of an extensive field trial and some comments on the continued work with respect to signal processing and evaluations. 


\section{SCENARIOS}

A total of seven urban scenarios categorized by its military objectives were developed:

- Intelligence gathering in an area of potential future conflict (global)

- Supporting special forces and conventional forces operation

- Conventional forces attacking a city, aiming to occupy it (focused intelligence, support attackers, support during attack)

- Battle damage assessment after urban fighting

- Avoiding IED attack

- Detection of prepared ambush by insurgents

- Detection and tracking of mobile targets

Each scenario is defined by its military objective, the campaign objective and the description of the operation, including the Area of Interest (AOI) and its features, the required assets and operation time.

Six of the scenarios are static urban scenarios and one is dynamic. Static scenarios were developed for intelligence gathering, Special Forces operations, conventional forces operations, Improvised Explosive Device (IED) detection, insurgents operations and mobile target detection. One dynamic scenario was developed for the tracking of moving military assets.

The main objective of the intelligence gathering scenario is the collection of intelligence about the landscape and the civilian and military activities. Personnel and vehicles were located at specific positions in the trial area. Soldiers were using different uniforms and weapons and took different positions (i.e. standing, lying) on different surfaces (i.e. concrete, grass), both in and outside shadows.

The Special Forces scenario focuses on the collection of detailed information about target, surroundings and on sensor support of the attacking force during an operation. The conventional forces scenario includes the attacking of a city, the defense of a city and the assessment of urban warfare damage. For the assessment of the battle damage, vehicles and surfaces were covered in sand or ashes, tents were collapsed to simulate building damage and corpses were simulated by lying soldiers. The insurgent scenario demonstrates the detection of an ambush prepared by insurgents. Simulated were ambushers in the open field, with horizontal coverage (e.g. behind a vehicle) and with both horizontal and vertical coverage (e.g. under a roof). Also obstacles were placed.

The scenario for IED detection focuses on the detection of the placement of an IED and the detection of an IED after placement.

The scenario of mobile target detection focuses on the detection of vehicles before and after relocation. Therefore different types of vehicles were located inside and outside the base and moved to different locations in between overpasses.

The dynamic scenario focuses on the (re)discovery and tracking of vehicles and personnel. To simulate this combination, vehicles and troops moved between different locations. By changing positions while being obscured from view behind buildings when moving, the (re)discovery was simulated.

\section{THE FIELD TRIAL}

The field trial was carried out during the period June to the beginning of July in a largely rainy Zeebrugge urban and harbor area, shown in Fig. 1. 


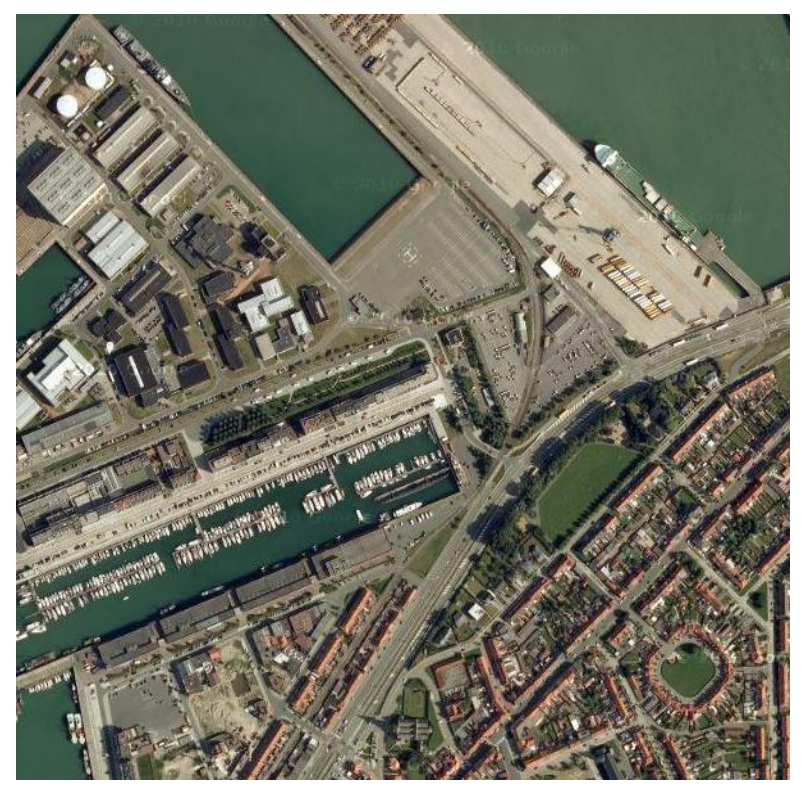

Figure 1. Zeebrugge harbor area (Google maps)

To add realism to the DUCAS campaign, static and dynamic military operations were simulated using actual military assets in the Area Of Interest (AOI) during the campaign. The operations were developed in cooperation between military and scientific participants. The Belgium armed forces conducted the operations at the Zeebrugge naval base during the campaign.

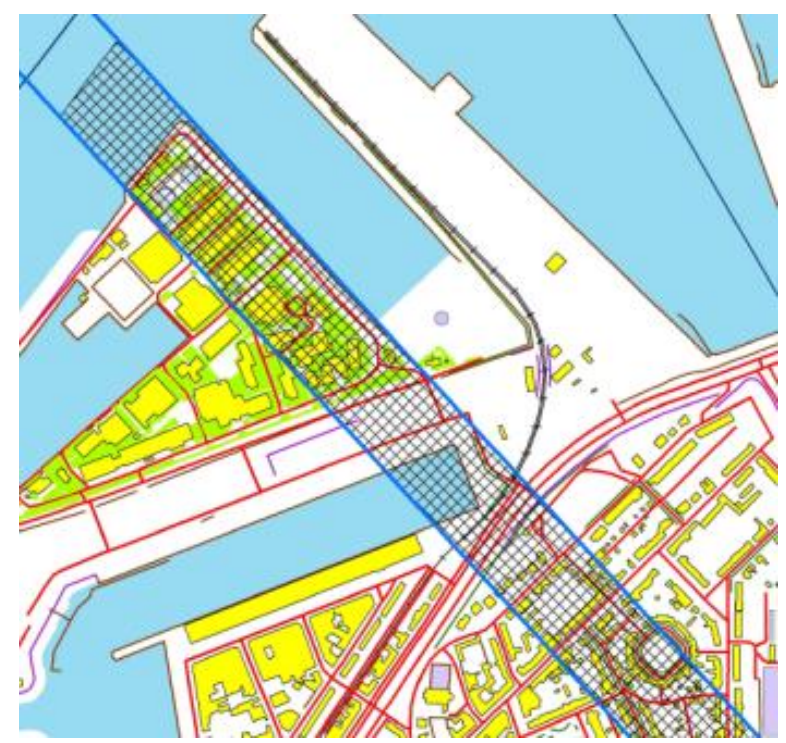

Figure 2. Primary area of interest for the airborne sensors.

Fig. 2. shows an example of the AOI for a specific scenario. The area of interest includes both the harbor and the downtown city. For each military scenario, relevant sensor tasks were identified, that potentially can be carried out by airborne sensors, with emphasis on tactical imaging sensors of various kinds. The scenario arrangement involved several hundred people in complex activity patterns. All this resulted in high demands on trial management and logistic 
coordination in order to assure the realization of the trial procedures. More than a dozen different types of vehicles and dozens of other objects were used in the trial.

\section{DATA COLLECTION}

A large database was obtained from the trial that can serve a host of applications. This database covers the whole chain from detailed surface measurements to the synoptic perspective of targets in varying backgrounds. This allows the study of patterns and interactions and serves the effort to parameterize observations. Another view is the temporal variation, both of specific surfaces and materials and of large areas.

Spectral imaging in the visible and near infrared wavelength region (associated with reflected sunlight) was performed during daytime. The thermal infrared spectral region, associated with the self-emission due to the temperature of the objects, was observed during both daytime and one night-time test. Sensing in the thermal infrared gives both day and night capability although the target-to-clutter ratio varies strongly with the solar irradiance.

Airborne data were obtained using hyperspectral imaging sensors in the visible, near infrared, short wave infrared and the long wave thermal infrared spectral region. The spectral resolution varied between 3 and $8 \mathrm{~nm}$ except for the LWIR spectral region where it was closer to $100 \mathrm{~nm}$. The ground sampling distance varied between 0.2 and $1.0 \mathrm{~m}$.

For classification and identification of targets in the visible spectral region, high spatial resolution sensors with a ground sampling distance of the order of $5 \mathrm{~cm}$ were used. In the midwave infrared spectral region, a ground sampling distance of $0.3 \mathrm{~m}$ was obtained. Prior to the trial, the area was mapped using a lidar.

The spectral regions covered by the various instrumentations are shown in Fig. 3.

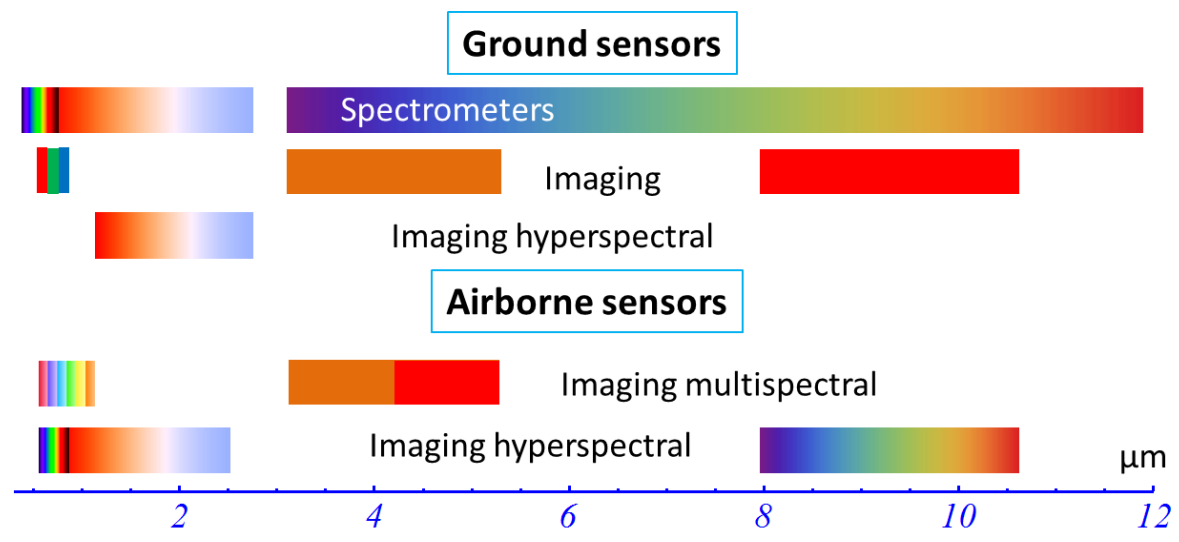

Figure 3. Overview of ground based sensors and airborne sensors.

The references for calibration of the data were of three types:

- Radiometric references for ground and airborne sensors.

- Position references for the airborne sensors.

- Radiometric references for ground truth sensors.

Radiometric calibration in the thermal infrared is performed using panel blackbodies both close to the sensor and as part of reference scene elements. Temperatures were selected between the low air temperature and hot solar heated background temperatures. Calibration activities were regularly performed. The reference area containing the various types of panels is shown in Fig. 4. 
To support target classification and change detection, as well as analytical modeling, atmospheric characteristics were monitored. Both the microphysics of the atmosphere and the radiative properties are of interest. The characteristics include the water vapor profile, temperature profile, aerosol content, wind speed and direction at ground level, solar irradiance and cloud coverage.

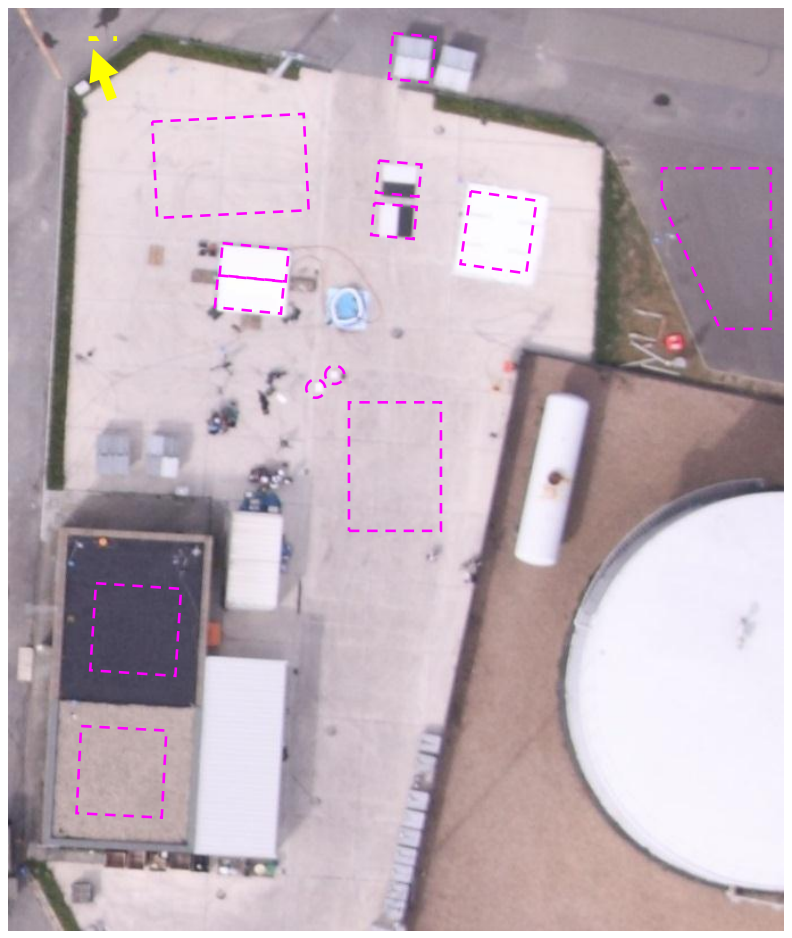

Figure 4. Reference panel area showing e.g. resolution edge targets for both the reflective and the thermal spectral domain. The temperature of the panels was carefully monitored.

\section{PROCESSING PROSPECTS}

There are a large number of issues that can be addressed using the data collected during the trial. The combined performance of high spatial resolution sensors and hyperspectral sensors is a signal processing challenge. The main design and evaluation areas that will be addressed from a signal processing point of view are:

- Phenomenology understanding

- Anomaly detection

- Signature detection

- Target and background classification

- Target tracking

- Change detection

Hyperspectral anomaly detection is a powerful target detection tool. In the urban environment anomaly detection will result in a large number of detected objects, and the technique hence have to be combined with other techniques such as classification or change detection for detection of anomalous changes. Hyperspectral signature detection and high resolution tracking are other techniques relevant to the urban environment.

For any given detector, the trade-off between probability of detection and probability of false alarm, i.e. the Receiver Operating Characteristics (ROC) must be considered. This must be combined with an analytical model forecasting system performance for system design and parameter trade off considerations. The urban environment poses considerable challenges and there is still a need to improve on the state-of-the art methods currently employed. 
A major challenge of using hyperspectral imagery operationally, e.g., for automatic target recognition, is that the acquired spectra depend also on external factors that are not intrinsic to the material or object one is interested in detecting. Examples of such factors are illumination variations, current and past weather conditions, atmosphere conditions, and ground geometry, e.g., if the object is in shadow or not. The trial provides the opportunity to model and estimate such phenomenology, with the aim to develop robust spectral matching methods that are invariant to the spectral variability due to external factors. For example, hyperspectral data have been collected over several days, so that variability over different weather conditions for a large number of materials can be studied and compared to the ground truth spectra. Objects were placed in shadow and open regions in a controlled way, so that the effect of shadow and illumination can be studied closely. With the help of the 3D model of the Zeebrugge base, light pathways and wall reflections can be modeled and studied. Finally, with help of the continuously monitored weather data, atmospheric modeling methods can be evaluated. In terms of method development, both accurate and simplified models and methods will be developed, with a focus on getting real-time spectral matching performance.

The combination of sensors can be optimized for any specific application. As an example, near infrared images were combined with midwave infrared images in order to detect shadows without using three-dimensional information. The result obtained for downtown Zeebrugge on a sunny day is shown in Fig. 5.

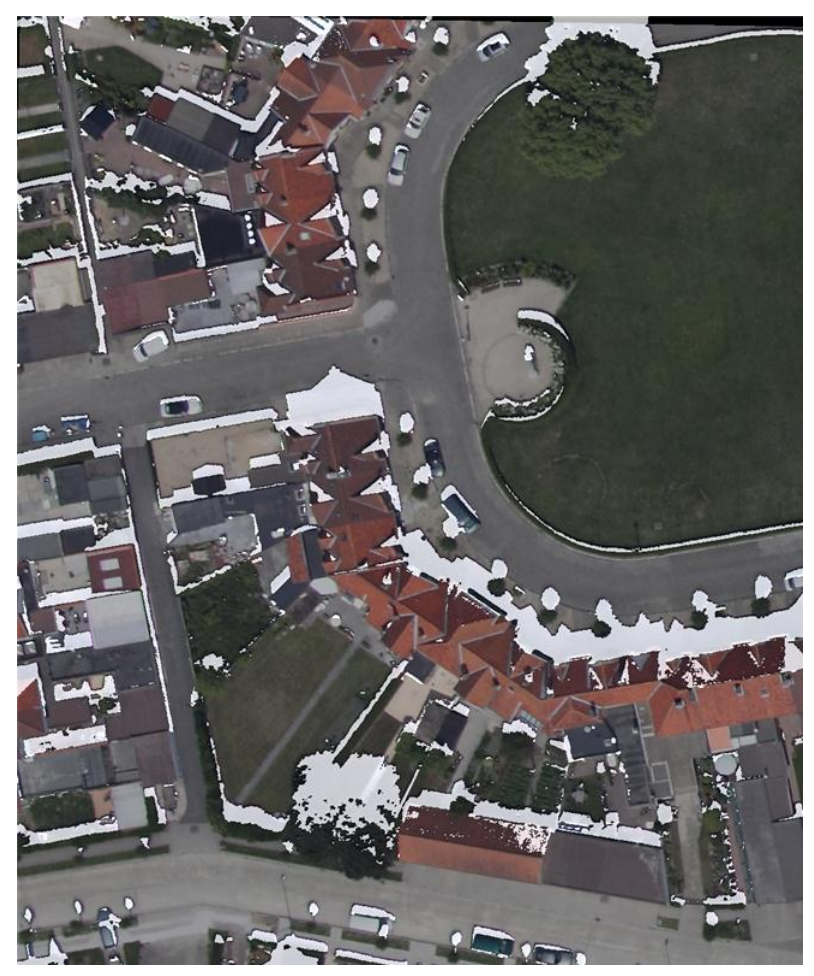

Figure 5. Estimated shadows from reflective and thermal imagery are shown in white for clarity, superimposed on a visible color image.

In Fig. 6, hyperspectral information in the visible and near infrared has been used to locate certain types of objects (red cars) based on novel constrained subspace spectral matching. 


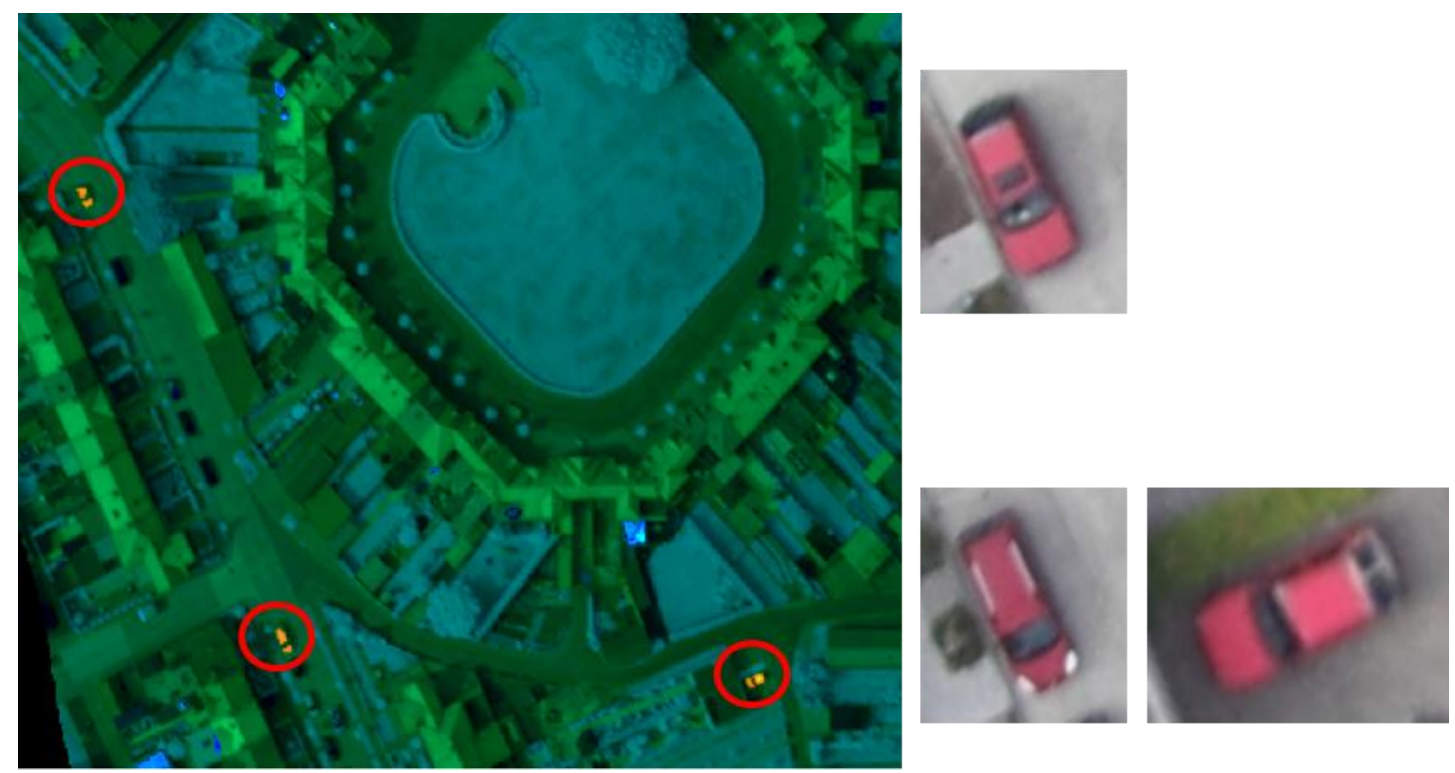

Figure 6. Detection of red cars in the city using constrained subspace spectral matching.

As another example we can show the case of detecting small changes in urban scenario due to vehicles/personnel movements. In this case, since a prompt response has to be given in near real time, the analyst can benefit from the use of combined sensors, detecting changes by means of algorithms applied to data and recognizing changes by means of high spatial resolution images, as shown in Fig. 7. In this example, it is shown that changes can be detected without the need of perfect alignment of recordings that are distorted due to irregular platform motion.

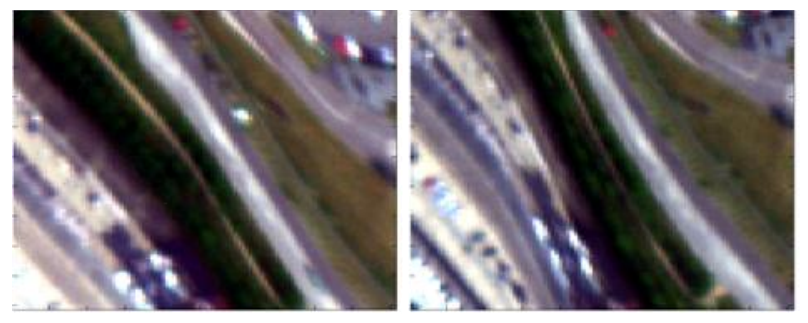

(a)

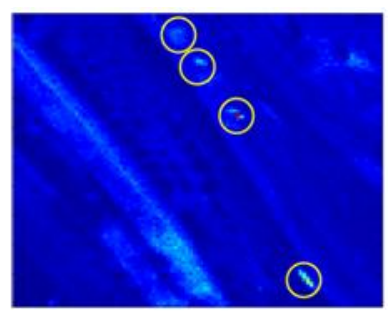

(b)

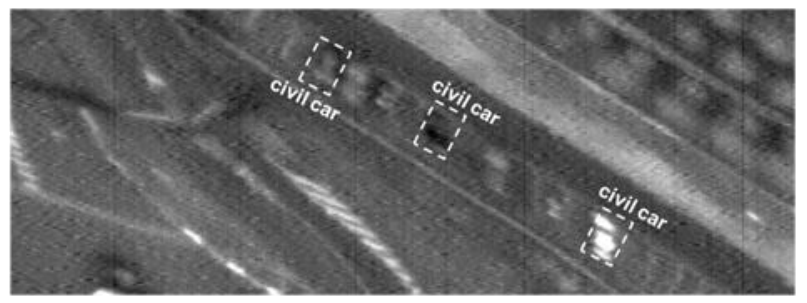

(c)

Figure 7. RGB display of hyperspectral data - Test Image on Left and Reference Image on Right (a) Local Co-Registration Adjustment Spectral Angle Mapping Algorithm Output (b) Detail of the scene - Panchromatic Image (c) 


\section{CONCLUSION}

Seven leading European research institutes have joined efforts to investigate the potential benefit of combined high spatial and spectral resolution airborne imagery for several defense applications in urban areas. An extensive field campaign was conducted at Zeebrugge in Belgium during June 2011. A detailed documentation of both the equipment and the measurements was obtained. Extensive sets of data were recorded. Preliminary assessments shows that useful data are available for addressing the issues set out at the beginning of the trial. The detailed ground truth data will also allow modeling of the scene and assess the capabilities of the airborne sensors. Specific signature features can be addressed in a systematic way.

The conditions to reach the objective of the project can therefore be considered to be good.

\section{REFERENCES}

[1] B. C., Forster, "An examination of some problems and solutions in monitoring urban areas from satellite platform", International Journal of remote Sensing, 6 (1), 139-151, 1985.

[2] G. F. Hepner, Houshmand, B., Kulikov, I. and Bryant, N., "Investigation of the integration of AVIRIS and IFSAR for urban analysis", Photogrammetric Engineering and Remote Sensing, 64 (8), 813-820, 1998.

[3] C. M., Chen, Hepner, G. F. and Forster, R. R., "Fusion of hyperspectral and radar data using the HSI transformation to enhance urban surface features", Photogrammetric Engineering and Remote Sensing, 58, 1930, 2003.

[4] C., Small, "Global Analysis of Urban Reflectance", International Journal of Remote Sensing, Article in press, 2004.

[5] D. L., Robie, «The air force needs new glasses - sensor requirements for urban operations », Each Air \& Space Power Journal, Sep, 2006.

[6] . « CEPA 8 - JP 8.10 Military applications of hyperspectral imagery »; X. Briottet, Y. Boucher, Dimmeler A., Malaplate A., Cini A., Diani M., Bekman H., Schwering P., Skauli T., Kasen I., Renhorn I., Klasen L., Gilmore M., Oxford D. ; WEAO Research News (the news letter for European Defence R\&T Cooperation, $\mathrm{N}^{\circ} 39,1$ st August 2005. 